\title{
EXPERIENCIAS PARA LA CAPACITACIÓN DE RECURSOS HUMANOS EN SALUD PARA LA ATENCIÓN INTEGRAL DE VÍCTIMAS DE VIOLENCIA EN UNA ZONA URBANO MARGINAL DE LIMA, PERÚ
}

\author{
Fausto Garmendia ${ }^{1,2, a, b}$, Alberto Perales ${ }^{1,3, b, c}$, Eva Miranda ${ }^{1, d}$, Pedro Mendoza ${ }^{1, b, ~ e, ~}$ \\ Walter Calderón ${ }^{1, e}$, Jorge Miano ${ }^{1, e}$
}

\begin{abstract}
RESUMEN
En el año 2003, en la Facultad de Medicina de la Universidad Nacional Mayor de San Marcos, se creó el Programa Permanente de Capacitación para la Atención Integral de las Víctimas de la Violencia, y viene realizando programas de capacitación de recursos humanos para la atención integral de las víctimas de violencia. En ese sentido, se consideró necesario desarrollar una metodología para profesionales de la salud, identificando sus necesidades de capacitación y sus condiciones de trabajo en lo relacionado a este tipo de atenciones. Es en ese contexto, que el año 2004, se delineó un estudio de línea de base en la Microrred de Salud Huaycán, al este de la ciudad de Lima; el cual comprendió diversas etapas con un enfoque multisectorial con el objetivo de identificar las necesidades de capacitación de los profesionales de salud, así como la evaluación del soporte logístico y administrativo para el desarrollo de actividades de capacitación a diversos niveles. En el presente artículo se expone, de modo sucinto, los procedimientos y principales resultados encontrados. Se evidenció que la población de Huaycán se encontraba afectada por las secuelas de la violencia política; no obstante, los servicios de salud tienen recursos limitados para realizar una adecuada atención de estas personas, emerge la necesidad de capacitación en varias esferas; existen condiciones administrativas y logísticas para efectuar estas actividades. Consideramos que esta metodología facilitará construir productos e instrumentos para una adecuada y específica capacitación para la atención integral en salud a las víctimas de la violencia.
\end{abstract}

Palabras clave: Violencia; Atención integral de salud; Capacitación de recursos humanos en salud; Perú (fuente: DeCS BIREME).

\section{EXPERIENCES IN THE TRAINING OF HEALTH HUMAN RESOURCES FOR THE INTEGRAL CARE OF THE VICTIMS OF VIOLENCE IN A SUBURBAN AREA OF LIMA, PERU}

\begin{abstract}
In the year 2003, in the Faculty of Medicine of the Universidad Nacional Mayor de San Marcos, the Permanent Program of Training for the Integral Attention of the Victims of Violence was created, has been training human resources for the comprehensive health care to victims of violence. In this sense, we was considered necessary to develop a methodology for health professionals, identifying their training needs and the conditions under how they work. It is in this context, that the year 2004, a base line study was delineated in the Microrred de Salud Huaycán, in the east of Lima city; that included diverse stages with a multisectorial approach with the aim to identify the training needs of the health professionals, as well as the evaluation of the logistic and administrative support for the development of training activities to diverse levels. In this paper, the procedures and principal results are exposed, in a succinct way. There was demonstrated that the population of Huaycán were affected by the sequels of the political violence; nevertheless, the health services have severe limited resources to give appropriate health care to victims of violence. The health professionals require an intensive training on this issue. An adequate logistic and administrative conditions allowed to carry out an appropriate training program. We suggest that this methodology will facilitate to construct products and instruments for a suitable and specific training for the integral health care to the victims of the violence.
\end{abstract}

Key words: Violence; Comprehensive health care; Health human resource training; Peru (source: MeSH NLM).

\footnotetext{
Programa Permanente de Capacitación para la Atención Integral de las Víctimas de la Violencia (PPCAIVV), Facultad de Medicina, Universidad Nacional Mayor de San Marcos, Lima, Perú.

2 Instituto de Investigaciones Clínicas, Facultad de Medicina, Universidad Nacional Mayor de San Marcos, Lima, Perú.

3 Instituto de Ética en Salud, Facultad de Medicina, Universidad Nacional Mayor de San Marcos, Lima, Perú.

a Médico Internista y Endocrinólogo; b Doctor en Medicina; ' Médico Psiquiatra; d Enfermera, Magister en Educación; e Médico, Magister en Salud Pública.
}

Recibido: 13-10-10 Aprobado: 01-12-10 


\section{INTRODUCCIÓN}

En el año 2003, se creó en la Facultad de Medicina de la Universidad Nacional Mayor de San Marcos el Programa Permanente de Capacitación para la Atención Integral de las Víctimas de la Violencia (PPCAIVV) el cual, entre otras actividades, desarrolló un análisis colectivo a través de talleres de análisis de involucrados y de problemas; donde se contó con la participación de autoridades políticas y de salud, así como miembros de la Comisión de la Verdad y Reconciliación, miembros del Programa de Apoyo al Repoblamiento, miembros de la sociedad civil, docentes universitarios, profesores del Harvard Program in Refugee Trauma, miembros de la Agencia de Cooperación del Japón (JICA) y representantes de los grupos de personas afectadas por la violencia ${ }^{(1)}$.

Una de las principales conclusiones de ese análisis fue la urgente necesidad de capacitar al personal de salud que labora en el primer nivel de atención del Ministerio de Salud de Perú (MINSA), tendiente a lograr competencias para la atención integral a las personas afectadas por la violencia política y violación de sus derechos humanos, producidas en el conflicto armando interno en las últimas dos décadas del siglo XX. Para lograr ese objetivo y evaluar su impacto, se consideró necesario efectuar estudios de línea de base que permitan identificar sus necesidades de aprendizaje y capacitarlos en una forma más eficiente por medio de programas diseñados para tal propósito. Situación que ha derivado en la propuesta de una metodología práctica de trabajo, apropiada a este tipo de problemas y cuyas características se describen en el presente artículo. Más aun cuando nuestra hipótesis suponía que los profesionales de salud del primer nivel de atención no poseían los conocimientos y competencias para atender adecuadamente a las víctimas de la violencia, además de carecer de una metodología de capacitación apropiada para este fin.

La revisión bibliográfica nos vislumbró que los estudios de línea de base sobre violencia se encuentran más orientados a la obtención de información sobre prevalencia e incidencia de sus diversos tipos ${ }^{(2-5)}$, que a investigación en materia docente a nivel de los profesionales de la salud, que permita mejorar el proceso de enseñanza-aprendizaje en estos, a fin de alcanzar una atención integral a los afectados.

Es en ese contexto, que nuestra propuesta debería de contener las bases de una metodología específica y replicable, la cual contuvo la siguiente secuencia de acciones:

- Elegir el lugar donde se efectuará el estudio de base.
- Evaluar las características del medio social del lugar escogido.

- Coordinar con las autoridades e instituciones locales interesadas y comprometidas con el problema de violencia para facilitar las acciones.

- Identificar, ubicar y entrevistar a las víctimas para conocer las características de su enfermedad producto de las experiencias traumáticas sufridas, así como tener un acercamiento adecuado a sus necesidades en el marco de la atención integral de salud.

- Evaluar el estado actual del Sistema de Atención Primaria de Salud al cual pertenecen.

- Identificar las necesidades de capacitación de los profesionales de la salud de dicho sistema, en lo referente a la atención de estas personas.

- Conocer los requerimientos de orden logístico para llevar a cabo las actividades docentes.

\section{LA EXPERIENCIA EN HUAYCÁN DURANTE EL AÑO 2004}

\section{SELECCIÓN Y EVALUACIÓN DEL MEDIO SOCIAL}

Para el año 2004, la elección del lugar para realizar el estudio fue la Microrred de Salud Huaycán, parte de la Dirección de Salud (DISA) Lima-Este, sustentado en el hecho que su población había sufrido en forma intensa los efectos de la violencia política, al constituir una población con gran migración de desplazados por esta causa.

Huaycán se encuentra ubicada en el km 16,3 de la carretera central, en el distrito de Ate Vitarte, en el cono este de Lima Metropolitana, a los $12^{\circ} 01^{\prime} 18^{\prime \prime}$ latitud sur, y $76^{\circ} 54^{\prime} 57^{\prime \prime}$ longitud oeste, con una altitud que oscila entre 385 y $450 \mathrm{msnm}$. Se encuentra en una quebrada entre los cerros Fisgón y Huaycán, al este de las ruinas Incaicas que llevan ese mismo nombre. Tiene un área de $3066,4 \mathrm{~km}^{2}$, con una densidad poblacional de 48,78 y una ruralidad de 0,07 .

Es importante señalar que en el año 1983, se constituyen las asociaciones de vivienda "Andrés Avelino Cáceres" y "José Carlos Mariátegui", cuyos miembros eran personas desplazadas provenientes de Ayacucho y Huancavelica, entre otros - ubicados inicialmente en los distritos de Ate-Vitarte, El Agustino, La Victoria y Chosica - quienes de modo pacífico y organizado, ocupan estos terrenos eriazos el 5 de julio de 1984. Es así como la Comunidad Urbana Autogestionaria de Huaycán (CUAH) surge como producto de un Plan Especial de Habilitación Urbana (PEHUH), creado por la Municipalidad de Lima Metropolitana. 


\section{RED DE CONTACTOS Y COORDINACIONES}

Tras la selección, se construyó una red de contactos y coordinaciones entre los diferentes niveles de la DISA, gobierno municipal, representantes locales de la Iglesia Católica, representantes de la Policía Nacional del Perú (PNP), organizaciones interesadas en atender problemas sociales, organizaciones no gubernamentales (ONG), asociaciones de personas afectadas por la violencia, promotores de salud e informantes clave bilingües. Lo que nos permitió tener un gran apoyo para identificar y ubicar a las víctimas de violencia política residentes en ese lugar.

\section{UBICACIÓN E IDENTIFICACIÓN DE LAS VÍCTIMAS DE VIOLENCIA}

Se elaboró un mapa de distribución de la población total de Huaycán, que proyectada para el año 2004 alcanzaba a un total de 150068 (basado en lo sostenido por el Hospital MINSA del lugar). Con lo que logró ubicar a 108 familias afectadas por la violencia política. El principal lugar de procedencia de las familias desplazadas por esa condición, fue Ayacucho (39,8\%), en segundo lugar Huancavelica con $24 \%$ y Junín con $13,8 \%$. El $50,3 \%$ de los afectados es de sexo femenino, el número de hijos promedio por familia fue de 3,7 , con rango que va desde 1 a 10 , es importante remarcar que el $20,6 \%$ de las participantes eran viudas.

\section{PERFIL CLÍNICO DE LAS VÍCTIMAS}

Se evaluó el estado actual de salud de las víctimas, abordando el perfil clínico del impacto sufrido por las experiencias traumáticas, mediante atenciones clínicas - empleando la Décima Revisión de la Clasificación Internacional de las Enfermedades. Transtornos Mentales y del Comportamiento, $\mathrm{CIE}-10^{\left({ }^{(6)}\right.}$ - de los directamente afectados y de familiares seleccionados.

Se entrevistaron diez adultos, víctimas directas de la violencia terrorista (CIE 10: Z65.4). Todas fueron de sexo femenino, con bajo nivel de educación, con un promedio de edad de 50,1 años en un rango de 32 a 80. De ellas, nueve tenían patología psiquiátrica, cuyo perfil clínico abarca desde trastorno de estrés postraumático crónico en cinco de ellas (CIE 10: F43.1), episodios depresivos leve y moderado (CIE10: F32.0 y F32.1) en cuatro de ellas, trastorno mixto ansioso-depresivo en tres (CIE10: F41.2), así como, trastorno disociativo (de conversión) mixto (CIE10: F44.7) y trastorno psicótico agudo transitorio sin especificación (F23.9) pero con menor frecuencia. También se entrevistó a familiares de estas mujeres, donde dos de sus hijos fueron diagnosticados con trastorno depresivo leve con síntomas somáticos (CIE10: F32.01) y trastorno ansioso-depresivo (CIE10: F41.2).
Es importante acotar el hecho de que las víctimas directas muestran mínima conducta de búsqueda de ayuda profesional con relación a las consecuencias sufridas a causa de la violencia, situación que condiciona un subregistro importante en los registros de salud de esa comunidad. Además, es cardinal enfatizar la ausencia de medicación psiquiátrica básica al momento del desarrollo del estudio de línea de base. Por comunicación personal desde el área de psicología del Hospital Huaycán, conocemos que la atención actual es fundamentalmente, por violencia familiar, abuso sexual, trastornos depresivos y trastornos ansiosos.

Vale la pena mencionar, que en el aspecto ético, se aprecian deficiencias en el manejo de la relación médicopaciente. En promedio, el $60 \%$ de la consulta se gasta en tareas de llenado de formularios. Se observa, además, desconocimiento de técnicas simples de psicoterapia de apoyo emocional.

\section{DIAGNÓSTICO DE LA ATENCIÓN PRIMARIA DE SALUD}

Con una población de 150068 habitantes, Huaycán cuenta con tres establecimientos de salud: el Hospital Local Huaycán, el Puesto de Salud Señor de los Milagros y el Puesto de Salud Horacio Zevallos.

En el Hospital de Huaycán laboran 132 trabajadores, de los cuales $48(36 \%)$ son trabajadores nombrados. Se cuenta con 29 médicos y una psicóloga. Como fortaleza organizativa, es importante mencionar que el hospital es considerado sede de capacitación materno infantil del Ministerio de Salud, por lo cual hay una dinámica ya instalada de mejora continua, un buen nivel de protocolización de sus acciones y la disposición favorable para acoger otros programas y procesos de capacitación.

El perfil de morbilidad de Huaycán es concordante con el de otras zonas urbano-marginales de Perú, siendo la primera causa de consulta las infecciones agudas.

\section{DIAGNÓSTICO DE LAS NECESIDADES DE CAPACITACIÓN DE LOS PROFESIONALES DEL PRIMER NIVEL DE ATENCIÓN}

Subsiguientemente, se llevó a cabo un estudio descriptivo transversal con enfoque cuali-cuantitativo, que tenía como objetivo principal la identificación de las necesidades de capacitación de los profesionales de la salud de la microrred en mención, para proporcionar una apropiada atención integral a las víctimas de la violencia. A este nivel también se construyó, y en algunos casos se adecuaron, instrumentos y técnicas de recolección de información. Otro alcance importante en esta fase, 
es que el reconocimiento de sus necesidades permitirá sentar directrices motivacionales en base a ello.

Se identificó a 132 profesionales de la salud candidatos a participar en el proceso de capacitación. El $95 \%$ de ellos no cuenta con experiencia en la atención a personas afectadas por la violencia. Las necesidades de capacitación prioritarias se centran en el fortalecimiento de las competencias para realizar la prestación de servicios, que abarca las áreas de diagnóstico, tratamiento y recuperación. El 100\% de profesionales médicos tiene dificultades para realizar el diagnóstico del trauma agudo y crónico, la psicosis y el estrés postraumático y el $100 \%$ de enfermeras refiere no tener competencias para el diagnóstico de los problemas de salud mental relacionados con la violencia.

Consideramos que la totalidad de profesionales requieren ser capacitados en la atención protocolizada de personas afectadas por la violencia, el tratamiento farmacológico - exclusivo para médicos-, la medicina complementaria, la medicina alternativa, recuperación psicológica, tratamiento dirigido a la recuperación y a la reversión de los procesos patogénicos.

Existen necesidades de capacitación en el tratamiento destinado a estabilizar y apoyar a las víctimas y prevenir su deterioro. Un aspecto importante, señalado por los profesionales, es la necesidad de capacitación en las modalidades individual, grupal y familiar de la psicoterapia, así como en la consejería y apoyo emocional orientando hacia la conducta de búsqueda de ayuda y solución de problemas. Otra necesidad expresada se centra en el manejo del dolor psicológico, el proceso de duelo de la víctima de violencia, la familia y comunidad, así como en el manejo de los instrumentos, mecanismos y relaciones para establecer un abordaje holístico con un importante componente espiritual.

En ese sentido, con el propósito de favorecer la referencia oportuna, los profesionales requieren capacitarse en los factores de riesgo individuales, los factores de riesgo sociales y la identificación de signos y síntomas de alarma correspondientes.

Otra necesidad sentida por la mayoría de los profesionales está relacionada con la recuperación integral, cuya base es el programa de reparaciones en salud. Las necesidades de capacitación en las áreas preventiva y promocional deben de orientarse hacia los derechos ciudadanos y a promocionar la salud mediante estrategias a nivel individual, estrategias relacionales, iniciativas de base comunitaria y estrategias basadas en la sociedad.

Un aspecto no menos importante, es la capacitación para realizar investigaciones operativas que permitan mejorar la calidad de la atención, además, fortalecer las habilidades educativas centrándose en la metodología de educación de adultos y la planificación, desarrollo y evaluación de procesos educativos.

En el área actitudinal, las necesidades de capacitación se orientan hacia la sensibilización para la reconstitución de las redes de soporte social y para el fortalecimiento de las capacidades hacia el desarrollo personal y social de los afectados por la violencia. Así mismo, en la capacidad de liderazgo, de problematización de la práctica.

De acuerdo con la percepción de las personas afectadas por la violencia, las necesidades de capacitación de los trabajadores de salud se centran en las relaciones interpersonales, relación proveedor-usuario, en este caso la relación con la persona víctima de violencia, derechos humanos y atención con enfoque intercultural.

El análisis del escenario político institucional y las características funcionales de los trabajadores de salud permiten afirmar que existe viabilidad para el desarrollo de la estrategia de capacitación. Sin embargo, se debe tener en cuenta algunos factores tales como el sistema de trabajo, el horario, los ambientes de capacitación y un sistema de sostenibilidad de la propuesta.

Si bien durante el estudio se identificó 132 profesionales de la salud como base potencial para el proceso de capacitación, es prudente realizar la selección final de acuerdo con criterios objetivos; asimismo, debe de incluirse a personal de la oficina de capacitación, a promotores de salud y a personas afectas por la violencia que habitan en la microrred bajo estudio. ${ }^{1}$

\section{SOPORTE LOGÍSTICO Y ADMINISTRATIVO}

Finalmente, se evaluó las necesidades de orden logístico para llevar a cabo todas las actividades mencionadas anteriormente. Es primordial tener en cuenta la distancia y accesibilidad como base para el desarrollo de las diligencias correspondientes, en ese sentido, el distrito de Huaycán se encuentra a 16 km de Lima, tomando la carretera central, por lo que la movilización por vía terrestre es simple y fluida, por ello no es necesario contar con alojamiento nocturno en esa localidad. Las actividades pueden efectuarse viajando todos los días y regresando a Lima para pernoctar. En Huaycán existen todas las

Condición que fue considerada cuando se realizó el proceso de capacitación, como continuación del presente proyecto. 
facilidades para atender las necesidades elementales tanto de docentes como de alumnos. En el Hospital de Huaycán existe un local adecuado para la capacitación de 40 personas, así como los equipos necesarios para la presentación de ayudas audiovisuales.

\section{DISCUSIÓN}

La violencia es un problema de salud pública de ámbito mundial, por lo que se la ha considerado como un problema global de salud. En el año 2000 la Organización Mundial de la Salud (OMS), estimó que en el mundo se producían alrededor 1600000 muertes al año por causa de la violencia ${ }^{(8)}$. En la historia republicana de Perú, el conflicto armado interno de 1980 al 2000 constituye el episodio de mayor violencia, en el que la Comisión de la Verdad y Reconciliación (CVR) estimó la muerte o desaparición de cerca de 70000 personas ${ }^{(9)}$ y de más un millón de personas afectadas directamente por la violencia política y otras formas de violencia, cuyas consecuencias y secuelas se mantienen hasta la actualidad.

Es, así mismo, preocupante que a partir de esas dos décadas se han desarrollado otras formas de violencia relacionadas con el pandillaje, narcotráfico, alcoholismo, entre otras; las que alteran la salud de las personas de muy diversas formas. De acuerdo con el informe de la CVR y con estudios de línea de base efectuados por nuestro equipo de investigación, los profesionales de la salud del primer nivel de atención no están suficientemente preparados para atender adecuadamente a las víctimas de violencia ${ }^{(9,10)}$. De allí que haya nacido la iniciativa de capacitarlos, se suma a esto el hecho que no se cuenta con el número suficiente de especialistas fuera de la capital de la república.

Como ya se mencionó en párrafos previos, en el año 2003, la Facultad de Medicina de la UNMSM emprendió la tarea de formar ${ }^{(11)}$ y capacitar recursos humanos en salud para la atención integral de las víctimas de violencia mediante la creación del PPCAIVV que, desde entonces, ha efectuado múltiples estudios de línea de base y actividades de capacitación. En ese sentido y ante la necesidad de efectuar actividades docentes en forma desconcentrada y no existir una metodología apropiada para estos fines, el PPCAIVV se abocó a la implementación de una metodología racional y científica que permitiese capacitar profesionales de la salud de acuerdo con el perfil patológico de las víctimas, a sus necesidades de aprendizaje y a las características del medio laboral y social en el que trabajan.
De esta manera, es cardinal el establecimiento de los contactos y coordinaciones a nivel multisectorial, se debe considerar a las autoridades de salud, los gobiernos regionales, los gobiernos municipales, representantes de las iglesias, PNP, ONG, universidades, representantes de las organizaciones de víctimas de violencia y otras organizaciones; que serían apoyo significativo para obtener información sobre la situación social de las localidades donde se debe efectuar la capacitación.

Lo realizado en la población de Huaycán durante el año 2004, nos muestra una población que se encuentra aún muy afectada por los estragos de la violencia, con importante sintomatología psiquiátrica, además de desintegración de la estructura familiar, pérdida de la participación en la sociedad por indiferencia y desánimo. No obstante, es categórico el hecho de que la gran mayoría del enorme contingente de desplazados afectados por la violencia no había desarrollado la correspondiente conducta de búsqueda de ayuda hacia las instituciones de salud. Al respecto, Perales identifica algunos factores explicativos ${ }^{(12)}$ :

- Un número de víctimas directas expresan, aún angustiadas, que Sendero Luminoso ${ }^{2}$ tiene ojos y oídos por todas partes y que, de asistir al hospital, pueden ser identificados por sus datos personales $y$, en consecuencia, eliminados.

- Otros dicen que aquellos que se atrevieron a consultar recibieron en los centros de salud un trato inadecuado y fueron, con frecuencia, discriminados. Vale decir que el personal de salud no les ofreció un trato humanitario acorde con las circunstancias.

- Muchos otros originarios de la sierra del país, quechua hablantes y con otros patrones culturales, no consideran que las secuelas ocasionadas por la violencia terrorista sufrida, sea enfermedad. Señalan que es, más bien, sufrimiento, y, como tal, no corresponde al hospital o a los médicos tratarlo. En otras palabras que la estructuración social de enfermedad no se ha producido en estas personas, cargadas de un alto nivel de sufrimiento moral que los orientó, con más frecuencia, a desarrollar conducta de búsqueda de ayuda hacia las iglesias en pos de sosiego espiritual.

- Del mismo modo, algunos solicitan ayuda a los agentes de la medicina tradicional.

Por otro lado, se ha podido apreciar la negativa asociación entre la violencia política y el desarrollo de otros problemas sociales tales como alcoholismo, violencia familiar, explotación sexual, aparición de pandillas juveniles y aumento

\footnotetext{
2 Es una organización terrorista de tendencia ideológica maoísta originada en Perú en la década de 1980, que sembró violencia durante dos décadas en el país.
} 
de la pobreza, elementos que deben ser considerados en forma global para la búsqueda de las mejores soluciones.

Si bien es cierto que la organización estructural y funcional de los servicios de salud tiene patrones semejantes por pertenecer a una misma institución - el MINSA - se aprecian diferencias notables en algunos aspectos, debido a carencia de recursos humanos; situación que es imprescindible conocer de antemano para efectuar los requerimientos oportunos para una buena atención de salud.

En el caso particular de la microrred Huaycán, se observa que el personal de salud requiere de una amplia y total capacitación en el campo de la violencia, en gran parte debido a la carencia de formación en sus respectivas casas de estudios.

No resulta factible el efectuar una buena capacitación si no se conocen los aspectos logísticos relacionados, como la accesibilidad y disposición de ambientes y equipos, situación que ha sido abordada en el caso de la población bajo estudio.

\section{CONCLUSIONES}

La aplicación del diseño metodológico utilizado en la microrred Huaycán, nos muestra que es un procedimiento sencillo y práctico, que permite un conocimiento más amplio y cabal, no solo las necesidades de capacitación de los profesionales de salud involucrados, sino de todo el contexto que los rodea. El registro sistemático de estos procedimientos facilita una adecuada atención integral en salud a las víctimas de la violencia política en comunidades de desplazados.

\section{Financiamiento}

Realizado con soporte de la Facultad de Medicina de la Universidad Nacional Mayor de San Marcos, la Agencia de Cooperación del Japón y el Ministerio de Salud de Perú.

\section{Conflictos de Interés}

Los autores expresan no tener conflictos de interés con la publicación de este artículo.

\section{REFERENCIAS BIBLIOGRÁFICAS}

1. Garmendia F, Perales A, Miranda E, Mendoza P, Calderón W, Miano J, et al. Estudios de línea de base como metodología para una mejor capacitación para la atención integral de las víctimas de violencia. Libro de Resúmenes del I Congreso Peruano de Educación Médica. Asociación Peruana de Facultades de Medicina. Lima, Perú. 30 de noviembre al 3 de diciembre, 2005. p.20.
2. Garmendia F, Perales A, Miano J, Miranda E, Watanabe E. Derechos humanos y atención primaria en salud a personas traumatizadas por la violencia en el Perú. Talleres de Análisis de Involucrados y de Problemas. Cusco, Lima e Ica. Lima: Facultad de Medicina, Universidad Nacional Mayor de San Marcos; 2003.

3. Caballero T. Análisis de la violencia familiar: propuesta de una metodología para el trabajo preventivo de los trabajadores sociales. Santiago de Cuba: Universidad de Oriente; 2002.

4. Bardales O. Estado de las investigaciones sobre violencia familiar y sexual en el Perú 2001-2005. Lima: MINDES; 2006.

5. Iniciativas para la Identidad y la Inclusión. Resultados preliminares del estudio de la línea de base del Programa Prevención de la violencia y desarrollo integral de las y los jóvenes en México y Centroamérica. Documento de trabajo. México DF: COFADEH; 2007.

6. Instituto Estatal de las Mujeres de Nuevo León. Compendio de estudios sobre violencia familiar contra las mujeres. Monterrey: IEMNL; 2008.

7. Organización Mundial de la Salud (OMS). CIE-10. Décima revisión de la Clasificación Internacional de las Enfermedades. Transtornos mentales y del comportamiento. Madrid: Meditor; 1992.

8. Organización Panamericana de la Salud. Capítulo 1. La violencia, un problema mundial de salud pública. En: OPS. Informe mundial sobre la violencia y la salud. Washington DC: OPS; 2003

9. Comisión de la Verdad y Reconciliación. Informe Final de la Comisión de la Verdad y Reconciliación, 2003. Lima: CVR; 2003.

10. Garmendia F, Perales A, Miranda E, Mendoza P, Calderón W, Miano J. Informe Final del Estudio de línea de base de la micro red Huaycán, Programa Permanente de Capacitación para la Atención Integral de las Víctimas de la Violencia. Lima: Facultad de Medicina, Universidad Nacional Mayor de San Marcos; 2004.

11. Miranda E, Garmendia F, Perales A, Mendoza P, Miano J, Calderón W. Evaluación de los planes curriculares y sílabos de las asignaturas de la Facultad de Medicina de la UNMSM, sobre la atención integral de salud a personas afectadas por la violencia. An Fac Med (Lima). 2009;70(4):273-76.

12. Perales A. Situación de la Violencia y de la Formación de Recursos Humanos en Salud en el Perú. En: Violencia en Latinoamérica. Actas del Seminario Taller Latinoamericano "Formación y Capacitación de Recursos Humanos en Salud para la Atención Integral de Víctimas de la Violencia y Violación de Derechos Humanos". Lima: UNMSM/Harvard Program in Refugee Trauma/JICA; 2004. p. 206-15.

Correspondencia: Fausto Garmendia

Dirección: Paseo de la República N. 3691-401B, Lima 27,

Perú.

Teléfono: (51) 998-673-812

Correo electrónico: faustogarmendia@yahoo.com 